\title{
NIH Transformative High Resolution Cryo-Electron Microscopy Program
}

Paula Flicker ${ }^{1}$, Mary Ann $\mathrm{Wu}^{1}$ and Houmam Araj ${ }^{2}$

${ }^{1}$ National Institute of General Medical Sciences, Bethesda, Maryland, United States, ${ }^{2}$ National Eye Institute, Bethesda, Maryland, United States

Cryo-electron microscopy (cryo-EM) is a method used to image frozen biological molecules without the use of structure-altering dyes or fixatives or the need for crystallization. This approach provides accurate models of the molecules and supports a greater understanding of biological function. Recent advances in cryo-EM detector and image processing technologies have made it possible for scientists to obtain detailed structures of many biological molecules, at resolutions as low as $2 \AA$, that cannot be obtained readily using other methods. Despite the emergence of cryo-EM as a powerful high-resolution imaging method, its use is hampered by inadequate access to equipment and a limited workforce.

The National Institutes of Health $(\mathrm{NIH})$, through the trans-NIH Common Fund Program, supports programs to broaden access to cryo-electron microscopy and to build a skilled workforce. The Transformative High Resolution Cryo-Electron Microscopy program has funded three national cryo-EM service centers to provide access to the technology and is supporting the development of cryo-EM training curricula to build a skilled workforce. The centers are established with six-year awards to provide scientists with access to state-of-the-art cryo-EM technology and training, from sample preparation to collection of high-resolution data and computational analysis. User access for data collection or cross training is provided through a peer reviewed open-access application process. Additional four grants fund curriculum development to train novice and more experienced users on cryo-EM technology and theory. The curricula will include online video lectures, software and e-books, 3-D animations, and interactive virtual reality. To further advance access to and expertise in the usage of cryo-ET, NIH is following the model of cryo-EM service centers to establish a national network of service centers for cryoET in 2020. Access the NIH Common Fund web site to find out more: https://commonfund.nih.gov/CryoEM.

References

https://commonfund.nih.gov/CryoEM. 